\title{
The Parameter Optimization Design Of The Naked Eye Stereoscopic Display With Slit Grating
}

\author{
Jun liu ${ }^{1}$, and Lintao $\mathrm{Liu}^{2}$ \\ ${ }^{1}$ University of Electronic Science and Technology of China, Chengdu , 610000, china \\ ${ }^{2}$ University of Electronic Science and Technology of China, Chengdu , 610000 , china
}

\begin{abstract}
Based on the principle of slit grating free stereoscopic display, an optical system model was established and the system was simulated with tracepro optical design software. Through the analysis of the distribution fringe pattern of light through narrow slit, a method for increasing the visual area and reducing crosstalk is presented. The optimal design of the slit grating free stereo display is realized.
\end{abstract}

\section{INTRODUCTION}

With the improvement of people's living standard and people's continuous pursuit of quality of life. The traditional $2 \mathrm{~d}$ image display can not meet the needs of people, and $3 \mathrm{~d}$ display technology has attracted more and more people's interest to become the main research direction. Compared with the $2 \mathrm{~d}$ display technology, the $3 \mathrm{~d}$ display technology can make the image not limited to the plane of the screen[1], and can show the image with the deep feeling. It enables the observer to directly see the motion, depth and distribution of the objects in the image to obtain more comprehensive information.

At present, the mainstream 3D image viewing in the market is wearing polarized glasses, but wearing polarized glasses for a long time can cause nausea, dizziness and other discomfort. Eyeglass polarized glasses free stereo display technology without any feeling of nausea, dizziness, discomfort, and that the user can view in stereo image when his eyes from the eyes, and the watch range is bigger, is an important research object of the current display technology[2]. The naked eye stereoscopic display mainly consists of slit grating stereoscopic display and lens grating display. With the advantages of simple structure, easy implementation and low cost, the narrow gap grating stereoscopic display is the most likely product to be commercialized..

Slit grating stereo display there is a serious loss, viewing area such as resolution jump and image mutual crosstalk between the flaws, the principle of some colleges and universities and scientific research units have more in-depth research to solve these problems[3]. However, there are no studies on the theory of pixels in the free stereoscopic display based on slit grating.

\section{The three-dimensional display principle of slit grating}

When the human eye is watching the real world, the image of the object on the retina of the person's eyes is slightly different, and the difference is the parallax of the eyes. This disparity is fused by the visual cortex of the brain, creating a three-dimensional sense.

Raster free stereo display makes the use of human eyes parallax characteristic design. The principle is shown in figure 1, placing a grating plate in front of the LCD screen. After adjusting grating and the distance of the liquid crystal panel, left, right through the grating slit watch to write by the parity of pixels of left and right eye parallax images, is just to see the right image in the right eye and left eye just to see the left eye image[4]. This allows our brains to merge the images of the left and right eyes, and then we can see images in the brain that are stereoscopic.

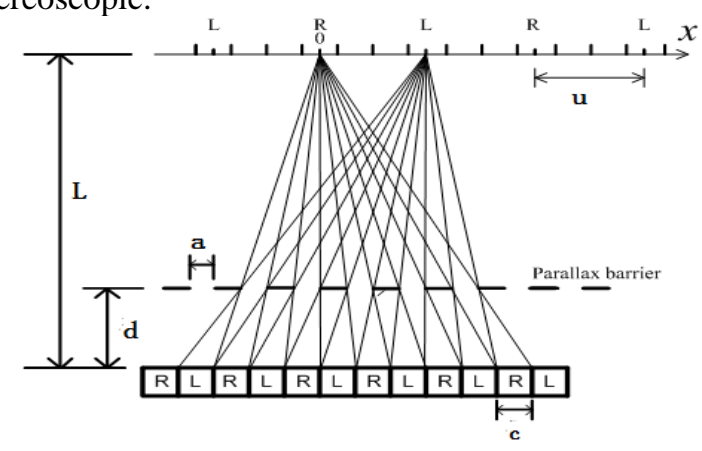

Figure 1 The principle of free three-dimensional display of slit grating

The graph above shows the geometrical structure model of the slit grating free stereo display. The spacing between the eyes of the human eye is u, the width of the slit and baffle is a, the distance from the grating to the LCD screen is $d$, and the pixel width is c. The distance between the distance to the LCD screen and the geometric relationship between parameters are as follows: 


$$
\begin{array}{r}
a=\frac{c \times u}{c+u} \\
d=\frac{c \times L}{c+u}
\end{array}
$$

According to the geometric relationship of formula (1) and (2), the grating type free stereo display has been able to display the stereo image correctly. But the figure 1 is a kind of simplified model of optical system, the sub pixel line light source model as a point light source, and ignored each pixel unit is the direction of propagation of the beam of a real random. Therefore, in order to improve the display quality of the free stereoscopic display, the system parameters must be optimized.

\section{Theory and optimization analysis.}

The area in which the stereoscopic display can form a stereoscopic display is just a part of the screen, which is called the visible area, with two viewpoints as an example, as shown in figure 2 . Where, $u$. Represents the left and right dimensions of the visible area, affecting the range of the viewer's eye movement. Figure 2 illustrates the visual areas staggered in the horizontal direction, due to the randomness of the light propagation direction, the adjacent independent visual range, viewers will see from other light anaglyph, referred to as the stereo image crosstalk[5]. The existence of crosstalk greatly reduces the stereo display effect, so crosstalk reflects the advantages and disadvantages of stereoscopic vision. This paper defined the following method to measure Crosstalk: the two viewpoints of stereo display of left channel Crosstalk, for example, in a dark room when the viewer in the left eye view position, let the left image shows all white and right like display completely black, record $\mathrm{L}_{\mathrm{Lwb}}$ luminance information; Let the right image display all white while the left image shows full black, recording brightness information $\mathrm{L}_{\mathrm{Lbw}}$. ; Let left and right image display all black, record brightness information $\mathrm{L}_{\mathrm{Lbb}}$. Thus, the Crosstalk formula of the left eye channel is calculated.

$$
C_{L}=\frac{\mathrm{L}_{\mathrm{Lbw}}-\mathrm{L}_{\mathrm{Lbb}}}{\mathrm{L}_{\mathrm{Lwb}}-\mathrm{L}_{\mathrm{Lbb}}} \times 100 \%
$$

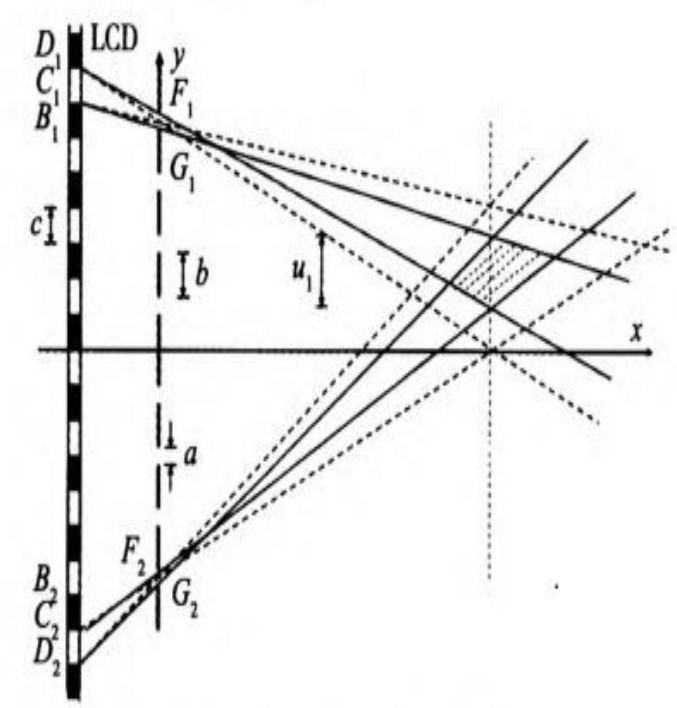

Figure 2 Slit grating can be viewed as a geometric model. In order to minimize the chance of the human eye to see crosstalk, when designing the parameters of slit grating, it is necessary to increase the left and right dimensions of the visual area.

See figure 2, The horizontal direction of the LCD screen is $\mathrm{m}$, There are $\mathrm{n}$ slits on the slit grating.According to figure 2, the coordinate system is established. The parallel Y-axis of the slit grating is placed on the Y-axis. The LCD screen is parallel to the $\mathrm{Y}$-axis and is placed in the $\mathrm{d}$ of the slit grating. The coordinates of each point are shown in table 1.[6]

Table 1 The coordinates of each spot

\begin{tabular}{|c|c|c|}
\hline spot & $\mathbf{x}$ & $\mathbf{y}$ \\
\hline C1 & $-d$ & $(2 n+1) c$ \\
\hline D1 & $-d$ & $2 n c$ \\
\hline F1 & 0 & {$[n(a+b)+a] / 2$} \\
\hline G1 & 0 & {$[n(a+b)-a] / 2$} \\
\hline C2 & $-d$ & $-(2 n-1) c$ \\
\hline D2 & $-d$ & $-2 n c$ \\
\hline F2 & 0 & $-[n(a+b)+a] / 2$ \\
\hline G2 & 0 & $-[n(a+b)+a] / 2$ \\
\hline
\end{tabular}

The visible region of the right eye can be seen in the stereo image, which is composed of linear C1F1, D1G1, $\mathrm{C} 2 \mathrm{~F} 2$ and D2G2. According to the coordinates of table 1, the equations of linear C1F1, D1G1, C2F2 and D2G2 are known:

$$
\text { C1F1: } \quad y=\frac{[n(a+b)+a] / 2-(2 n+1) c}{d} x+[n(a+b)+a] / 2
$$




$$
\begin{aligned}
& \text { D1G1: } y=\frac{[n(a+b)-a] / 2-2 n c}{d} x+[n(a+b)-a] / 2 \\
& \text { C2F2: } \quad y=\frac{-[n(a+b)-a] / 2+(2 n-1) c}{d} x-[n(a+b)-a] / 2 \\
& \text { D2G2: } y=\frac{-[n(a+b)+a] / 2+2 n c}{d} x+[n(a+b)+a] / 2
\end{aligned}
$$

The distance of the horizontal direction of the viewpoint region is calculated according to the difference between the intersection point of the line D1G1 and $\mathrm{D} 2 \mathrm{G} 2$ and the intersection of the line $\mathrm{C} 1 \mathrm{~F} 1$ and $\mathrm{C} 2 \mathrm{~F} 2$.

$$
u_{1}=\frac{(b-a) c}{2 c-b-a}
$$

As can be seen from formula (8) and figure 2, u1 changes with the change of slit a. If the slit a decreases and the $\mathrm{u} 1$ becomes larger, the loss rate of the light can be increased due to the blocking effect of the grating plate. When the viewpoint number is determined, the period of the slit grating is fixed, and the increase of slit a can effectively reduce the photoenergy loss rate and increase the display brightness, but $\mathrm{u} 1$ is obviously smaller. Known from the analysis of the above, the naked eye slit grating stereo display, display brightness and a contradiction between the three-dimensional effect of relationship, in the LCD back light source intensity is uncertain, usually of contradiction is the basic idea of display brightness can accept under the condition of optimum design based on stereo vision demand slit a value.

\section{Simulation results and discussion.}

In this paper, we use Tracepro optical design software to simulate the free naked eye stereoscopic display of slit grating. Tracepro provides a powerful ray tracing feature. It can be used to get the spatial distribution of the random transmission through the grating. According to the previous analysis, the parameters of the main correction system in the optimization design are the width $\mathrm{a}_{1}$ of the transparent part of the slit grating. For comparison with the experimental data, the pixel width $\mathrm{c}$ is the actual value of the 15-inch LCD screen that I used in the experiment. The distance of the light is $885 \mathrm{~mm}$. The distance between the slit grating and the LCD screen is $2 \mathrm{~mm}$. During the simulation, the value of a is gradually changed near the theoretical value. The left and right size $\mathrm{u}_{1}$, crosstalk value $\mathrm{C}_{\mathrm{L}}$ and light energy loss rate were calculated.

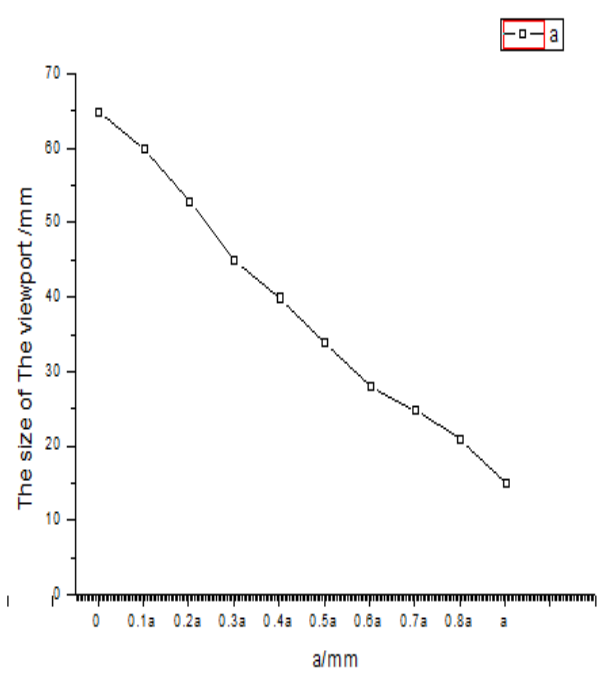

Figure 3 The left and right dimensions of the visual area vary with $\mathrm{a}_{1}$

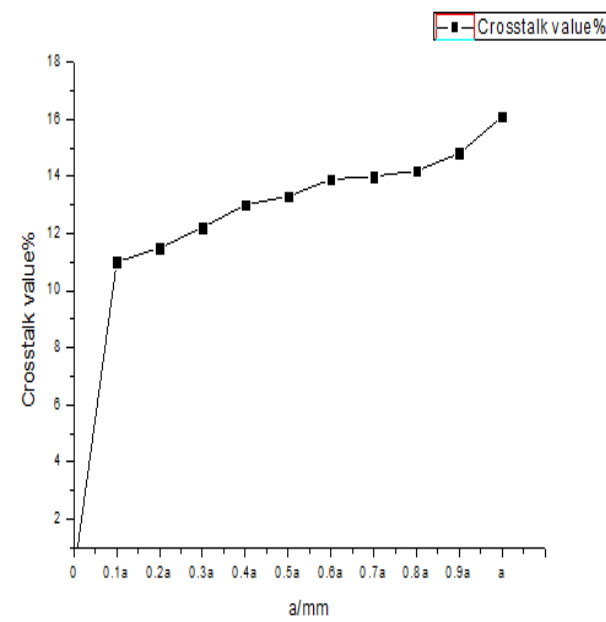

Figure4 Crosstalk value $C_{L}$ varies with a1.

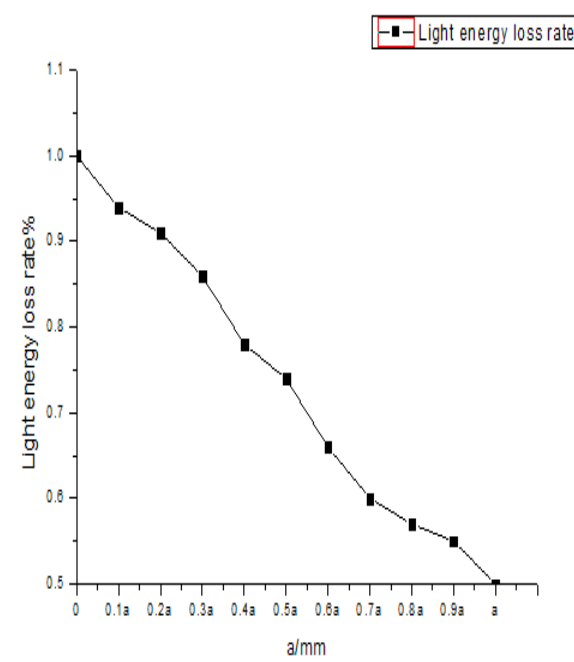

Figure5 The light energy loss rate varies with $\mathrm{a}_{1}$

As can be seen from FIG. 3, the experimental data and theoretical analysis are basically the same, and the increase of the left and right size $u_{1}$ is reduced from 65 $\mathrm{mm}$ to $6 \mathrm{~mm}$. The larger the $\mathrm{u}_{1}$ value is, the viewer can have a certain range of activities, which is conducive to viewing. If the left and right size of the viewing area is 
too small, the position requirement of the viewer is more strict, which makes the display stereo vision performance decrease. As can be seen from figure 4 the crosstalk value $\mathrm{C}_{\mathrm{L}}$ increases with the increase of $\mathrm{a}_{1}$. This graph shows that in actual viewing, there will also be crosstalk between the best position images. In the experiment, we found that when The value of $\mathrm{C}_{\mathrm{L}}$ is greater than $15 \%$, and crosstalk seriously affects the stereoscopic display effect. It is shown that the appropriate reduction of the value of slot $a_{1}$ can effectively increase the left and right size of the visual area, and also improve the crosstalk problem between images. However, as can be seen from figure 5, if the value of $a_{1}$ is smaller, the greater the loss rate of light energy will be, resulting in a severe decrease in the brightness of the stereoscopic display, resulting in visual fatigue of the viewer. In combination with the above three graphs, the following experimental data can be obtained: when $a_{1}=a$, the theoretical value of $a$ is obtained, $\mathrm{u}_{1}=6 \mathrm{~mm}, \mathrm{C}_{\mathrm{L}}=16.5 \%$, and the light energy loss rate is 0.5 , obviously $\mathrm{u}_{1}$ is too small, $\mathrm{C}_{\mathrm{L}}$ is too big.In order to ensure the brightness, the loss rate of the light energy cannot be greater than $0.6, \mathrm{a}_{1}$ can only be greater than 0 . $7 \mathrm{a}$ at the same time, to obtain good visual effects, the $\mathrm{C}_{\mathrm{L}}$ value must be controlled under $15 \%$, and $\mathrm{a}_{1}$ can only be less than $0.9 \mathrm{a}$. Through the above analysis, in general, The value of the $a_{1}$ is $0.8 \mathrm{a}$ is suitable. At this point, $\mathrm{u}_{1}=15 \mathrm{~mm}, \mathrm{C}_{\mathrm{L}}=14.2 \%$, light energy loss rate 0.57 . Compared with the theoretical value of the mouth, $\mathrm{u}_{1}$ increased by $9 \mathrm{~mm}$ and $\mathrm{C}_{\mathrm{L}}$ decreased by $2.3 \%$, although the light energy loss rate increases by 0.07 , but the display brightness will not be too low, such stereoscopic display of the visual effect to achieve the best.

\section{Conclusions}

In this paper, the influence of the change of width $\mathrm{a}_{1}$ on the aperture of the slit grating is studied on the influence of the left and right size and crosstalk value of the free stereoscopic display area of the slit grating. Results show that the display backlight source intensity of certain circumstances, the width of the slit grating pervious to light part a of the according to actual needs, considering crosstalk size and image visual impact, in slightly less than the reference value of formula (1) calculate the appropriate selection of scope. The experimental results show that, in general, $\mathrm{a}_{1}=0.9 \mathrm{a}$, that is, the aperture width of the grating is reduced to $90 \%$ of the reference value calculated by formula (1), and the visual effect of the stereoscopic display is the best.

\section{References}

1. Liang yunyun, Deng shanxi, Yang yongyue. The optical structure and setting of the LCD screen in the stereoscopic display [J]. Liquid crystal and display, , 2005, 20(6): 544-548.

2. Wang aihong, Wang qionghua. Overview of raster free stereoscopic display [J]. Modern display, 2009 (10): $12-17$.

3. Dodgson N A. Autostereoscopic 3D Displays[J]. Ccomputer, 2005, 38(8):31-36.

4. Wen jiangtao, Hu yuehui, Lu guoqiang, etc. Study on the shadow problem of grating stereo display device [J]. Liquid crystal and display, , 2007, 22(3): 310-314.

5. Wang Q H,Zhao W X,Tao Y H,et al.Stereo viewing zone in parallax barrier based autostereoscopic display[J]. Optik, 2010, 121(22):2008-2011

6. Qin laughs, Luo jianli. Free stereo display technology and its development [J]. Journal of Chinese image graphics, 2009. 14(10) : 19341941. 